\title{
A precision analysis of camera distortion models
}

\author{
Zhongwei Tang, ${ }^{1}$ Rafael Grompone von Gioi, ${ }^{2}$ Pascal Monasse, ${ }^{1}$ and Jean-Michel Morel $^{2}$ \\ ${ }^{1}$ LIGM, UMR 8049, École des Ponts, UPE, Champs-sur-Marne, France \\ ${ }^{2}$ CMLA, ENS-Cachan, Cachan, France
}

\begin{abstract}
This paper addresses the question of identifying the right camera direct or inverse distortion model permitting a high subpixel precision fit to real camera distortion. Five classic camera distortion models are reviewed and their precision compared for direct or inverse distortion. By definition, the three radially symmetric models can only model a distortion radially symmetric around some distortion center. They can be extended to deal with non-radially symmetric distortions by adding tangential distortion components, but still may be too simple for very accurate modeling of real cameras. The polynomial and the rational models instead miss a physical or optical interpretation, but can cope equally with radially and nonradially symmetric distortions. Indeed, they do not require the evaluation of a distortion center. When requiring high precisions, we found that the distortion modeling must also be evaluated primarily as a numerical problem. Indeed, all models except the polynomial involve a non-linear minimization which increases the numerical risk. The estimation of a polynomial distortion model leads instead to a linear problem, which is secure and much faster. We concluded by extensive numerical experiments that, although high degree polynomials were required to reach a high precision of $1 / 100$ pixels, such polynomials were easily estimated and produced a precise distortion modeling without over-fitting. Our conclusion is validated by three independent experimental setups: The models were compared first on the lens distortion database of the Lensfun library by their distortion simulation and inversion power; second by fitting real camera distortions estimated by a non parametric algorithm; and finally by the absolute correction measurement provided by photographs of tightly stretched strings, warranting a high straightness.
\end{abstract}

Index Terms-distortion measurement, camera calibration

\section{INTRODUCTION}

The pinhole camera model is widely used in computer vision applications because of its simplicity and its linearity in terms of projective geometry [14]. But real cameras deviate from the ideal pinhole model. The main geometric deviation is a lens geometric distortion [3], possibly complicated by a deviation from planarity of the CCD shape. Thus an accurate camera distortion correction is the first step towards high precision 3D metric reconstruction from photographs. With the steady progress in lens quality and camera resolution, highprecision 3D reconstructions become feasible. But they require in turn higher camera distortion precisions than those provided by classic methods. The object of this paper is to investigate the validity of distortion models at the light of precision requirements increased by two or three orders of magnitude. This increased accuracy requires a new methodology for evaluating distortion models. Five models are studied and evaluated in the paper: the radial [3], division [10], FOV [9], polynomial (such as bicubic [15]), and rational [7], [13] models. They rely on several different hypotheses on the underlying distortion model. Clearly their precision depends on which model hypothesis is valid. Radially symmetric models are very precise for radially symmetric distortions. They can be extended to treat non-radially symmetric distortions by adding tangential components. The polynomial model and the rational model impose the fewer constraints on the distortion, to the cost of an increased number of model parameters. Thay way they can cope with radial and non-radial symmetric distortions as well. Among them the polynomial is the only linear model. Its estimation is a simple matrix inversion. All the other models require a somewhat complex non-linear minimization. We shall see that the linearity of the polynomial model reduces its numerical risk and makes its modeling precision closer to $1 / 100$ pixels for realistic camera distortions.

It could be argued that a correct model should be based on physical measurements on systems of lenses. Surprisingly enough, there is little physical background for the distortion models in the literature. According to Weng et al. [30], lens distortion can be decomposed into three effects: radial distortion, decentering distortion and thin prism distortion. Nevertheless, it is only marginally based on a physical background, and it is not clear that the real distortion precisely follows this model. In fact, the final distortion is the result of the cumulated effects of a complex lens system, of the camera geometry, and of the (not perfectly planar) shape of the image sensor. One is therefore led to figure out a flexible model with enough parameters to simulate any plausible distortion. In absence of a physical model, the model classification approach adopted here will be to look for models that actually cope with any realistic distortion, at a given precision.

Many works in the computer vision community assume that the distortion is radially symmetric around the center of the image, and the radial, division and FOV models are often used to simulate and inverse the radially symmetric distortion. Even though these models are not exactly (algebraically) invertible, the simulation and correction can be obtained at very high precision if the order of the model is high enough, as we will show in Section III] This explains why these models in the literature are used with the interchangeable roles of distorted points and undistorted points. For example, direct distortion models are used in global camera calibration [29], [32], [17], [30]. Yet, in most plumb-line methods [3], [9], [1], [2], [25], [23], [22], [6] or some pattern-free methods [26], [31], [10], [19], [28], [7], [21], [4], [16], the very same radial correction models are used without any fuss to inverse the distortion.

In practice, the distortion center is unknown and we do not even a priori know if the distortion is radially symmetric around a certain center. So the radially symmetric models 
with an arbitrarily fixed distortion center (typically, the image center) is not always a good model. The common practice is to fix the distortion center at some reasonable position and add tangential distortion components to the model. All the global camera calibration methods adopt this strategy by fixing the distortion center at the principal point. An alternative is to estimate the distortion center that makes the radially symmetric models fit optimally to the distortion. For example, Hartley and Kang [12] propose to estimate the distortion center under the assumption that the distortion is radially symmetric and monotone. Being more general, the polynomial model and the rational model are invariant to the distortion center and can be directly used to model the distortion without estimating any distortion center.

Our aim is to find models which are flexible and easy to use, producing small residual error no matter what reasonable distortion has been applied. Ideally we would like to test on all possible distortions of any existing camera lens. Since exhaustive testing is impossible, we resorted to the lens database of the LensFun library, which is known to contain the most complete freely available lens profiles. All the distortions provided by Lensfun are radially symmetric around the image center.

The question we raise here only makes sense within fixed accuracy bounds. This is a first caveat: any evaluation must be performed at a given precision. As a matter of fact, for off-the-shelf cameras, most distortion models perform well at a 1 pixel precision. The question is different when we aim at sub-pixel precisions. These precisions, up to $1 / 100$ pixels, are highly desirable when using cameras for stereovision or photogrammetric tasks. Indeed, in stereovision a determining factor is $b / h$, the ratio of baseline to average depth. A high ratio improves accuracy of depth determination by triangulation, but makes automatic image matching more difficult because of the significant viewangle change. On the contrary, a low ratio eases the point-matching step but requires sub-pixel estimation to avoid a strong quantization of recovered depths. The latter case is advisable for automatic processing, hence the need for highly accurate distortion correction.

The other caveat is that, although distortion models reflect a model of the optical lens, the real distortion must actually involve the whole system lens plus CCD. There is no way to guarantee that a CCD is absolutely flat, or exactly perpendicular to the optical axis. This explains why the camera distortion modeling remains, after all, an empirical question where no physical argument can be final. The ultimate decision is numerical.

The various distortion models will be carefully compared on lens distortions in the Lensfun library, permitting to quantify the ideal attainable precision. Then, the same models will be compared on their capacity to fit to real camera distortions (estimated by a non-parametric algorithm [11]). Finally, the distortion correction accuracy by each model will be evaluated by using the plumb-line approach, with photographs of tightly stretched strings, warranting a high straightness, and giving absolute measurements of the correction quality [27]. In short, there will be three different numerical validations of our conclusions.
This paper is organized as follows. Section $\Pi$ reviews five classic distortion models. Their power to simulate and inverse distortions are evaluated in Section III by synthetic experiments. Section IV and $\mathrm{V}$ describe the experiments performed on real cameras. Section $\mathrm{VI}$ sums up the lessons learned from these experiments.

\section{Distortion AND CORRECTION MODELS}

We denote by $\left(x_{u}, y_{u}\right)$ the coordinates of an undistorted point as would be observed by an ideal pinhole camera. Due to the lens geometric distortion, this point will be observed at coordinates $\left(x_{d}, y_{d}\right)$. The distortion is modeled by a function $f$ that transforms undistorted to distorted coordinates,

$$
x_{d}=f_{x}\left(x_{u}, y_{u}\right) \quad y_{d}=f_{y}\left(x_{u}, y_{u}\right) .
$$

A correction model $g$ performs the inverse transformation,

$$
x_{u}=g_{x}\left(x_{d}, y_{d}\right) \quad y_{u}=g_{y}\left(x_{d}, y_{d}\right) .
$$

A particularly interesting case is when the function $f$ or $g$ shows radial symmetry relative to a fixed distortion center $\left(x_{c}, y_{c}\right)$. In that case we obtain a compact formulation using radial coordinates $\bar{x}_{u}=x_{u}-x_{c}, \bar{y}_{u}=y_{u}-y_{c}, \bar{x}_{d}=x_{d}-x_{c}$ and $\bar{y}_{d}=y_{d}-y_{c}$; then, the distortion can be expressed as the transformation of the undistorted radius $r_{u}=\sqrt{\bar{x}_{u}^{2}+\bar{y}_{u}^{2}}$ to the distorted radius $r_{d}=\sqrt{\bar{x}_{d}^{2}+\bar{y}_{d}^{2}}$.

We start by reviewing the most current models. To treat both directions in a neutral way, we will write the models as transforming from coordinates $\left(x_{1}, y_{1}\right)$ to $\left(x_{2}, y_{2}\right)$. When a model would be used as a distortion model, $\left(x_{1}, y_{1}\right)$ will correspond to $\left(x_{u}, y_{u}\right)$ and $\left(x_{2}, y_{2}\right)$ to $\left(x_{d}, y_{d}\right)$, and it is the opposite when used as a correction model.

The radial model displaces a point along its radial direction originating at the distortion center. The new radius $r_{2}$ is a function of the original radius $r_{1}$,

$$
r_{2}=r_{1}\left(k_{0}+k_{1} r_{1}+k_{2} r_{1}^{2}+\cdots\right) .
$$

The parameter $k_{0}$ represents a scaling and therefore does not introduce distortion. The scaled image is distorted by $k_{1}, k_{2}$, ... If all are positive, we have a pincushion distortion; if all are negative, a barrel distortion. Mustache distortion occurs if the signs are not the same ${ }^{1}$. Note that the distortion center $\left(x_{c}, y_{c}\right)$ is also a parameter of radial models.

The division model is obtained by simply putting the factor term $\left(k_{0}+k_{1} r_{1}+k_{2} r_{1}^{2}+\cdots\right)$ on the denominator,

$$
r_{2}=\frac{r_{1}}{k_{0}+k_{1} r_{1}+k_{2} r_{1}^{2}+\cdots} .
$$

In these models, high-order coefficients are needed to model extreme distortion in fish-eye lenses or other wide angle lens systems. A more sparse representation is obtained by parameterizing the distortion by the field of view (FOV). The only parameter of the FOV model is the field of view parameter $\omega$ :

$$
r_{2}=\frac{\tan \left(r_{1} \tan (\omega)\right)}{\tan (\omega)} 2^{2}
$$

\footnotetext{
${ }^{1}$ To be exact, it depends on the concavity or convexity (or their absence) of the polynomial on the right hand side of 3 over the extent of the image.

${ }^{2}$ This formula is slightly different from the original one proposed in [9] because we work in the normalized image domain and we consider $\omega$ as half of the field of view instead of the full field of view.
} 
The Taylor expansion of the FOV model around $r_{1}=0$ is

$$
r_{2}=r_{1}+\frac{\tan ^{2}(\omega)}{3} r_{1}^{3}+\frac{2 \tan ^{4}(\omega)}{15} r_{1}^{5}+\cdots
$$

So the FOV model is a radial model with odd terms around $r_{1}=0$. Note that the order- 0 scale coefficient is fixed to be 1 and all the other coefficients are coupled to $\omega$. So to model more complex distortions, the authors proposed to complete it

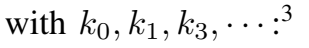

$$
r_{2}=\frac{\tan \left(r_{1} \tan (\omega)\right)}{\tan (\omega)}+r_{1}\left(k_{0}+k_{1} r_{1}+k_{3} r_{1}^{3} \cdots\right) .
$$

In the polynomial model the distortion is modeled as a polynomial in $x_{1}$ and $y_{1}$. For example, the third order (bicubic) polynomial model is

$$
\begin{aligned}
x_{2}= & a_{1} x_{1}^{3}+a_{2} x_{1}^{2} y_{1}+a_{3} x_{1} y_{1}^{2}+a_{4} y_{1}^{3}+a_{5} x_{1}^{2} \\
& +a_{6} x_{1} y_{1}+a_{7} y_{1}^{2}+a_{8} x_{1}+a_{9} y_{1}+a_{10}, \\
y_{2}= & b_{1} x_{1}^{3}+b_{2} x_{1}^{2} y_{1}+b_{3} x_{1} y_{1}^{2}+b_{4} y_{1}^{3}+b_{5} x_{1}^{2} \\
& +b_{6} x_{1} y_{1}+b_{7} y_{1}^{2}+b_{8} x_{1}+b_{9} y_{1}+b_{10},
\end{aligned}
$$

depending on the $(3+1) \cdot(3+2)=20$ parameters $a_{1}, \cdots, a_{10}, b_{1}, \cdots, b_{10}$. More generally, a polynomial model of order $n$ depends on $(n+1) \cdot(n+2)$ parameters. The rational function model is a quotient of two polynomials. A second order rational function model can be written as

$$
\begin{aligned}
& x_{2}=\frac{a_{1} x_{1}^{2}+a_{2} x_{1} y_{1}+\cdots+a_{5} y_{1}+a_{6}}{c_{1} x_{1}^{2}+c_{2} x_{1} y_{1}+\cdots+c_{5} y_{1}+c_{6}}, \\
& y_{2}=\frac{b_{1} x_{1}^{2}+b_{2} x_{1} y_{1}+\cdots+b_{5} y_{1}+b_{6}}{c_{1} x_{1}^{2}+c_{2} x_{1} y_{1}+\cdots+c_{5} y_{1}+c_{6}} .
\end{aligned}
$$

\section{Precision Evaluation}

We shall evaluate the distortion simulation and inversion power of the above mentioned models. Being a theoretical property of model families, the precision in both directions can be genuinely evaluated by synthetic experiments.

The test begins by synthesizing realistic distortions produced by a camera lens. Since it is impossible to exhaustively obtain all existing lens distortion profiles, we resort to the lens database of the public library LensFun, which inherits the database of the commercial software PTLens and has the most comprehensive freely available lens database (see Table 1 for a few lens examples. A complete lens list supported by Lensfun can be found at http://lensfun.sourceforge.net/lenslist/ We use version 0.3.2 of LensFun comprising more than 3500 models.). In Lensfun, the distortion is calibrated with some predefined models (see Table II), based on the matching points between two images taken by the same camera on the same focal length 4 The final calibrated distortion models in Lensfun are represented in the normalized image domain $[-1.0,+1.0] \times[-1.0,+1.0]^{5}$

\footnotetext{
${ }^{3}$ Originally the authors proposed to complete the FOV model with evenorder coefficients $k_{4}, k_{6}, \cdots$.

${ }^{4}$ See http://lensfun.sourceforge.net/calibration/ for the lens profile calibration procedure.

${ }^{5}$ Actually, the larger image dimension is mapped to $[-1.0,1.0]$ and the other dimension scaled so as to preserve the aspect ratio.
}

\begin{tabular}{|c|l|l|}
\hline lens maker & lens model & distortion model \\
\hline \multirow{3}{*}{ Canon } & Canon EF-S 10-22mm f/3.5-4.5 USM & ptlens \\
& Canon EF-S 18-55mm f/3.5-5.6 & ptlens \\
& Canon EF 24-105mm f/4L IS USM & ptlens \\
\hline \multirow{3}{*}{ Nikon } & Nikkor 12-24mm f/4G ED-IF AF-S DX & ptlens \\
& Nikkor 16-35mm f/4G ED-AFS VR & ptlens \\
& Nikkor 16-85mm f/3.5-5.6G AF-S ED VR DX & ptlens \\
\hline \multirow{3}{*}{ Sony } & Sony AF DT 16-105mm F3.5-5.6 & poly5 \\
& Sony DT 18-55mm F3.5-5.6 SAM SAL 1855 & poly3 \\
& Minolta/Sony AF DT 18-70mm F3.5-5.6 (D) & ptlens \\
\hline \multirow{3}{*}{ Olympus } & Zuiko Digital 7-14mm f/4.0 & ptlens \\
& Zuiko Digital 14-45mm f/3.5-5.6 & ptlens \\
& Zuiko Digital 40-150mm f/3.5-4.5 & ptlens \\
\hline \multirow{3}{*}{ Tamron } & Tamron 17-35mm f/2.8-4 Di LD & ptlens \\
& Tamron 17-50mm f/2.8 XR Di II LD & ptlens \\
& Tamron 18-200mm f/3.5-6.3 XR Di II LD & ptlens \\
\hline \multirow{3}{*}{ Pentax } & SMC Pentax DA 12-24mm F/4 ED AL IF & poly3 \\
& SMC Pentax DA 18-55mm f/3.5-5.6 & ptlens \\
& SMC Pentax DA 50-200mm f/4-5.6 DA ED & ptlens \\
\hline
\end{tabular}

TABLE I: Some lenses in LensFun used for synthetic tests. Note that our experiments are run on a complete list of lenses available in LensFun. Refer to LensFun website for more information. The distortion models referred here are written in Table II

\begin{tabular}{|l|l|}
\hline Model & Formulation \\
\hline ptlens & $r_{d}=r_{u}\left(1-a-b-c+c r_{u}+b r_{u}^{2}+a r_{u}^{3}\right)$ \\
\hline poly3 & $r_{d}=r_{u}\left(1-k_{1}+k_{1} r_{u}^{2}\right)$ \\
\hline poly5 & $r_{d}=r_{u}\left(1+k_{1} r_{u}^{2}+k_{2} r_{u}^{4}\right)$ \\
\hline
\end{tabular}

TABLE II: Models used to generate distortion in Lensfun.

According to each calibrated lens model in LensFun, we can synthetically generate distorted/undistorted point pairs in the normalized image domain. The simulation and correction precisions are then verified by identifying the best parameters through (1) and (2) respectively. In other words, both $\left(x_{u}, y_{u}\right)$ and $\left(x_{d}, y_{d}\right)$ are known in the synthetic tests, and the question is how well the distorted points $\left(x_{d}, y_{d}\right)$ can be approached by $f_{x}\left(x_{u}, y_{u}\right)$ and $f_{y}\left(x_{u}, y_{u}\right)$, and how well the ideal points $\left(x_{u}, y_{u}\right)$ can be approached by $g_{x}\left(x_{d}, y_{d}\right)$ and $g_{y}\left(x_{d}, y_{d}\right)$. We want to compute the coefficients of $f_{x}$ and $f_{y}$ by minimizing the difference between the observed distortion and the simulated distortion. The energy to be minimized can be written as

$$
C_{a}=\iint\left(f_{x}\left(x_{u}, y_{u}\right)-x_{d}\right)^{2}+\left(f_{y}\left(x_{u}, y_{u}\right)-y_{d}\right)^{2} \mathrm{~d} x_{u} \mathrm{~d} y_{u}
$$

Similarily, the energy to be minimized for estimating the coefficients of $g_{x}$ and $g_{y}$ can be written as

$$
C_{c}=\iint\left(g_{x}\left(x_{d}, y_{d}\right)-x_{u}\right)^{2}+\left(g_{y}\left(x_{d}, y_{d}\right)-y_{u}\right)^{2} \mathrm{~d} x_{d} \mathrm{~d} y_{d}
$$

In practice, we generated a total of $2 M$ pairs of distorted/undistorted points $\left\{\left(x_{d_{i}}, y_{d_{i}}\right),\left(x_{u_{i}}, y_{u_{i}}\right)\right\}_{i=1, \ldots, 2 M}$ uniformly distributed in the normalized image domain. Among these pairs, $M$ of them were used to estimate the parameter by minimizing the discrete energy

$$
D_{a}=\sum_{i=1}^{M}\left(f_{x}\left(x_{u_{i}}, y_{u_{i}}\right)-x_{d_{i}}\right)^{2}+\left(f_{y}\left(x_{u_{i}}, y_{u_{i}}\right)-y_{d_{i}}\right)^{2}
$$


and

$$
D_{c}=\sum_{i=1}^{M}\left(g_{x}\left(x_{d_{i}}, y_{d_{i}}\right)-x_{u_{i}}\right)^{2}+\left(g_{y}\left(x_{d_{i}}, y_{d_{i}}\right)-y_{u_{i}}\right)^{2}
$$

respectively. In order to verify the precision of the tested models, the estimated parameters were then used to compute the average residual error: $\sqrt{\frac{\hat{D}_{a}}{M}}$ and $\sqrt{\frac{\hat{D}_{c}}{M}}$ with $\hat{D}_{a}$ and $\hat{D}_{c}$ the energy computed on the other $M$ pairs of points, which were not used in the energy minimization. We used points on regular grids in $[-1,1]^{2}$ with $M=20 \times 20=400$.

The unknown parameters include the specific parameters of each distortion model and the distortion center $\left(x_{c}, y_{c}\right)$. This center $\left(x_{c}, y_{c}\right)$ is irrelevant for the polynomial model and the rational function model, both models being invariant to the distortion center. Yet for the other models, $\left(x_{c}, y_{c}\right)$ is an important parameter that must be accurately estimated to achieve precise distortion modeling [5], [12].

Linear or non-linear optimization methods are required to minimize the energy in (12) and (13). For the radial model (if the distortion center $\left(x_{c}, y_{c}\right)$ is known) and the polynomial model, the energies can be minimized by solving a linear system

$$
\mathbf{A k}=\mathbf{b},
$$

where $\mathbf{A}$ is a matrix containing the different powers for all the distorted (or undistorted) points, $\mathbf{k}$ is formed with the correction (or distortion) model coefficients, and $\mathbf{b}$ contains the undistorted (or distorted) coordinates. The set of model coefficients with least error is obtained by minimizing the residual $\|\mathbf{A k}-\mathbf{b}\|_{2}$, which results in

$$
\mathbf{k}=\left(\mathbf{A}^{T} \mathbf{A}\right)^{-1} \mathbf{A}^{T} \mathbf{b} .
$$

Since the synthetic tests can be done in the normalized image domain, the coefficient matrix $\mathbf{A}$ is usually well conditioned.

For all other models, a non-linear method must be used, even if $\left(x_{c}, y_{c}\right)$ is known. The minimization was performed by an incremental Levenberg-Marquardt (LM) algorithm which estimates the parameters in increasing order: The algorithm starts estimating the parameters of a second-order model; the result is used to initialize the model with the next higher order, and the process continues until the aimed order. The Jacobian matrix $\mathbf{J}$ required by LM was computed explicitly to make the algorithm efficient and more precise than with finite differences. We chose quite strict LM stopping criteria: each individual LM stops after 1000 iterations or when the energy does not decrease by more than $1 \mathrm{e}-10$. Even though this strategy was complex, it avoided some local minima and is safer than performing LM directly on the model at the aimed order.

Parameters initialization: For non-linear minimization, it is important to initialize the parameters with reasonable values. The common practice for the radial model and the division model is to initialize the 0th-order parameter $k_{0}$ to be 1 and all the other parameters to be 0 . The parameter $\omega$ in the FOV model is interpreted as half the field of view of the camera. For ordinary cameras, a typical value of $\omega$ is about $\frac{\pi}{6} \approx 0.52$, which can be can be used as initial value.
The initialization of the rational function model is more involved. As shown in [7], it can be solved linearly by using a "lifted process" technique. More precisely, $\left(x_{1}, y_{1}\right)$ and $\left(x_{2}, y_{2}\right)$ are related by a $3 \times 6$ matrix $\mathbf{A}$ :

$$
\begin{aligned}
\lambda\left(x_{2}, y_{2}, 1\right)^{T} & =\mathbf{A}\left(x_{1}^{2}, x_{1} y_{1}, y_{1}^{2}, x_{1}, y_{1}, 1\right)^{T}, \\
\mathbf{A} & =\left(\begin{array}{lllllll}
a_{1} & a_{2} & a_{3} & a_{4} & a_{5} & a_{6} \\
b_{1} & b_{2} & b_{3} & b_{4} & b_{5} & b_{6} \\
c_{1} & c_{2} & c_{3} & c_{4} & c_{5} & c_{6}
\end{array}\right) .
\end{aligned}
$$

A can be solved linearly by using at least 9 distorted/undistorted point pairs, similar to the classic homography estimation. This linear solution is used in the experiments to initialize the incremental LM algorithm, since it minimizes the algebraic error, which is not directly related to the geometric error we want to minimize and sometimes leads to undesirable result.

Distortion center initialization: The distortion center is a sensitive parameter for distortion models and should be accurately estimated to model or remove distortion precisely [5]. The common practice is to put the initial distortion center at the center of image. However, this is not always a safe initialization because the true solution can be away from the image center up to $10 \%$ of the image domain [5]. A more delicate approach was proposed [12] to linearly estimate the distortion center for radially symmetric models, which can be used as an initialization for our non-linear minimization. Based on the monotonicity assumption of the radially symmetric distortion, this method exploits the observation that the distorted points are obtained by moving the undistorted points away from (or to) the distortion center, similar to the motion of points seen by a camera moving forward toward/backward from a scene. So the distortion center plays the same role as the epipole of the fundamental matrix in a special situation, where the camera undergoes a pure translation between two views [14]. This observation leads to the following epipolar geometry [12]:

$$
\mathbf{x}_{d_{i}}^{T}[\mathbf{e}]_{\times} \mathbf{x}_{u_{i}}=\mathbf{x}_{d_{i}}^{T}\left([\mathbf{e}]_{\times} \mathbf{H}\right) \mathbf{x}_{c_{i}}=\mathbf{x}_{d_{i}}^{T} \mathbf{F} \mathbf{x}_{c_{i}}=0,
$$

with $[\mathbf{e}]_{\times}$the skew-symmetric $3 \times 3$ matrix representing the cross product by the distortion center $\mathbf{e}$. The undistorted points $\mathbf{x}_{u_{i}}$ are projected from the 3D points $\mathbf{x}_{c_{i}}$ on a planar calibration pattern through a pinhole camera: $\mathbf{x}_{u_{i}}=\mathbf{H x}_{c_{i}}$. The matrix $\mathbf{F}$ can be linearly computed from at least 7 pairs of $\left\{\mathbf{x}_{d_{i}}, \mathbf{x}_{u_{i}}\right\}$ correspondences [14]. In our case, $\mathbf{H}$ is the $3 \times 3$ identity matrix and we have simply: $\mathbf{x}_{d_{i}}^{T}[\mathbf{e}]_{\times} \mathbf{x}_{u_{i}}=0$. The distortion center e can then be computed as the right null vector of the matrix $[\mathbf{e}]_{\times}$, which can be solved by at least 7 distorted/undistorted point pairs, just like the fundamental matrix estimation in binocular stereo [14].

\section{A. Experiments with known distortion center}

We first consider the case where the distortion center is known. Then the radial model and the polynomial can be estimated by a matrix inversion, whereas all the other models require an incremental LM minimization. 
Simulation: Notice that the distortions are all generated by radially symmetric models, see Table [II So the radial model should be able to ideally simulate these distortions if it contains all the terms used in the distortion generation in Table II. Fig. 1a shows that the simulation precision is close to the machine limit except for the curve corresponding to the third-order radial model, which cannot simulate the distortions generated by higher order models (Table III). The division model can also simulate the distortion well. Even though this simulation is not exact, the precision increases with the order of the division model. In fact, a radially symmetric distortion can be written as

$$
\begin{aligned}
r_{2} & =r_{1}\left(k_{0}+k_{1} r_{1}+k_{2} r_{1}^{2}+\cdots\right)=\frac{r_{1}}{\frac{1}{\left(k_{0}+k_{1} r_{1}+k_{2} r_{1}^{2}+\cdots\right)}} \\
& =\frac{r_{1}}{\frac{1}{k_{0}}-\frac{k_{1}}{k_{0}^{2}} r_{1}+\frac{1}{k_{0}}\left(\frac{-k_{2}}{k_{0}}+\frac{k_{1}^{2}}{k_{0}^{2}}\right) r_{1}^{2}+\cdots},
\end{aligned}
$$

which means that the radially symmetric distortion can be well simulated by the division model with high enough order. For the FOV model, as we have shown before, since it has a high correlation with second order coefficient $k_{2}$ and has been completed by the other coefficients, it should also be able to simulate the radially symmetric distortion very precisely. However, in practice, both the division model and the FOV model estimation require a non-linear minimization and the resulting numerical risk explains why we obtained a less precise simulation in practice (Fig. $1 \mathrm{~b}$ and $1 \mathrm{c}$ ).

As for the polynomial model, it can simulate ideally the radially symmetric distortions composed of only even-order terms $r_{2}=r_{1}\left(k_{0}+k_{2} r_{1}^{2}+k_{4} r_{1}^{4}+\cdots\right)$ because

$$
\begin{aligned}
& x_{2}=x_{1}\left(k_{0}+k_{2} r_{1}^{2}+\cdots\right)=k_{0} x_{1}+k_{2} x_{1}^{3}+k_{2} x_{1} y_{1}^{2}+\cdots \\
& y_{2}=y_{1}\left(k_{0}+k_{2} r_{1}^{2}+\cdots\right)=k_{0} y_{1}+k_{2} x_{1}^{2} y_{1}+k_{2} y_{1}^{3}+\cdots
\end{aligned}
$$

are just two polynomials, with some monomials missing though. If odd-order terms also appear: $r_{2}=r_{1}\left(k_{0}+k_{1} r_{1}+\right.$ $\left.k_{2} r_{1}^{2}+\cdots\right)$, the polynomial model cannot ideally simulate the distortion. But the simulation precision increases with the order of polynomial model. Two polynomial models of adjacent orders (for example, the third-order and fourth order polynomials) have very similar performance due to the fact that the distorted/undistorted point pairs are almost radially symmetric and thus the odd-order terms in the polynomial model play a more important role to model such points. Also the error curve of the polynomial model is cut into three pieces due to the fact that the synthesized distortions can be classified into three groups: one group with only odd-order terms, one group with odd-order terms and one even-order term, and one group with odd-order terms and two even-order terms. These observations explain what we observe in Fig. 1e. In fact, if the radial model contains only odd terms, we observe the similar curves in Fig. 1d as in Fig. 1e The rational function model is more general than the polynomial model. So it should in theory improve the simulation power of the polynomial model (Fig. 11f).

The maximum residual error among the points, represented as dashed lines in the figure, is typically higher by a factor 10 than the average error. This shows that even the worst error remains low for most camera lenses.
Inversion: The correction power is the precision achieved by the distortion models to inverse the distortions in Lensfun. For any tested model, the inversion is not exact for the radially symmetric distortions in Lensfun. However, to inverse the distortion following $r_{2}=r_{1}\left(k_{0}+k_{1} r_{1}+k_{2} r_{2}^{2}+\cdots\right)$, we have

$$
\begin{aligned}
r_{1} & =\frac{r_{2}}{k_{0}+k_{1} r_{1}+k_{2} r_{1}^{2}+\cdots} \\
& =r_{2}\left(\frac{1}{k_{0}}-\frac{k_{1}}{k_{0}^{2}} r_{1}+\cdots\right) \\
& =r_{2}\left(\frac{1}{k_{0}}-\frac{k_{1}}{k_{0}^{2}} \cdot \frac{r_{2}}{k_{0}+k_{1} r_{1}+k_{2} r_{1}^{2}+\cdots}+\cdots\right) \\
& =r_{2}\left(\frac{1}{k_{0}}-\frac{k_{1}}{k_{0}^{2}} \cdot r_{2}\left(\frac{1}{k_{0}}-\frac{k_{1}}{k_{0}^{2}} r_{1}+\cdots\right)+\cdots\right) \\
& =r_{2}\left(\frac{1}{k_{0}}-\frac{k_{1}}{k_{0}^{3}} r_{2}+\frac{k_{1}^{2}}{k_{0}^{5}} r_{2}^{2}+\cdots\right)
\end{aligned}
$$

or

$$
\begin{aligned}
r_{1} & =\frac{r_{2}}{k_{0}+k_{1} r_{1}+k_{2} r_{1}^{2}+\cdots} \\
& =\frac{r_{2}}{k_{0}+k_{1} \cdot \frac{r_{2}}{k_{0}+k_{1} r_{1}+k_{2} r_{1}^{2}+\cdots}+\cdots} \\
& =\frac{r_{2}}{k_{0}+k_{1} r_{2}\left(\frac{1}{k_{0}}-\frac{k_{1}}{k_{0}^{2}} r_{1}+\cdots\right)+\cdots} \\
& =\frac{r_{2}}{k_{0}+\frac{k_{1}}{k_{0}} r_{2}-\frac{k_{1}^{2}}{k_{0}^{3}} r_{2}^{2} \cdots}
\end{aligned}
$$

which means that radially symmetric distortions are invertible by the radial model, the division model and the FOV model. The inversion is not exact and the precision increases with the order of the inversion model. In addition, with the same argument as before, the polynomial model and the rational function model can also be inverse of the radially symmetric distortion, in particular when the radially symmetric distortion is composed of only even-order terms. All the curves are shown in Fig. 1g 11] It was shown in [25] that the second order division model is superior to the radial of the same order; our experiments do not show such advantage for third or higher orders.

Today's digital cameras produce images of millions of pixels with a minimal order of magnitude of $1000 \times 1000$. We aim at an average precision about $10^{-2}$ pixel for images of such size. This is about $10^{-5}$ in the normalized image domain. All the models are able to simulate and correct the radially symmetric distortions provided by the Lensfun library at this precision if the order is high enough. In this restricted setting, they are all equivalent, notwithstanding the difficulty of minimization for some.

\section{B. Experiments with unknown distortion center}

In practice, the distortion center $\left(x_{c}, y_{c}\right)$ is unknown and we have to estimate it as well. The distorted/undistorted point pairs were generated in the same way as before except that the distortion center $\left(x_{c}, y_{c}\right)$ was unknown. We used the linear method proposed in [12] to first obtain an initial estimate of the distortion center, which was then refined with the 


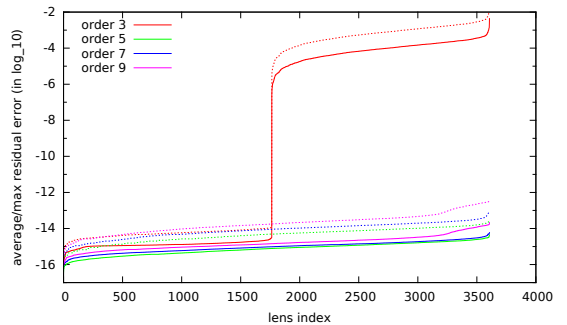

(a) radial model for distortion

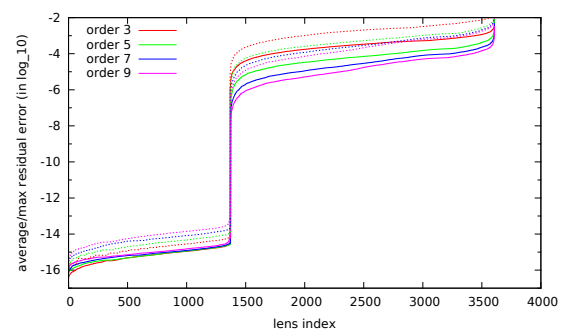

(d) odd-order radial model for distortion

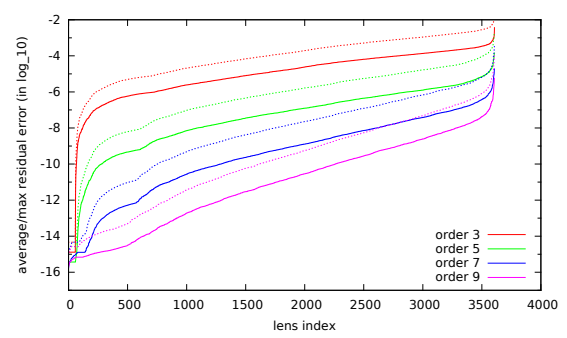

(g) radial model for correction

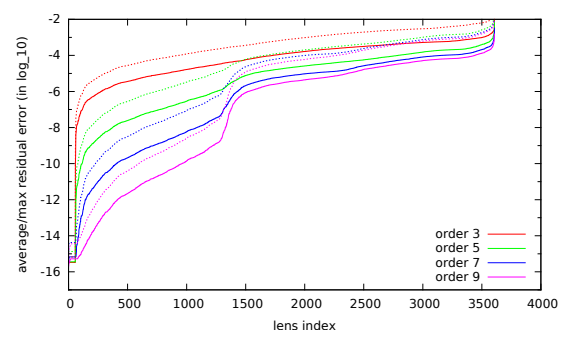

(j) odd-order radial model for correction

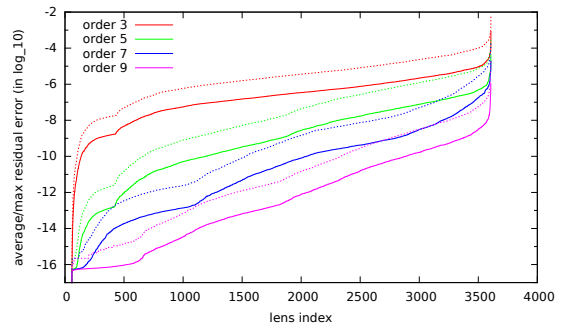

(b) division model for distortion

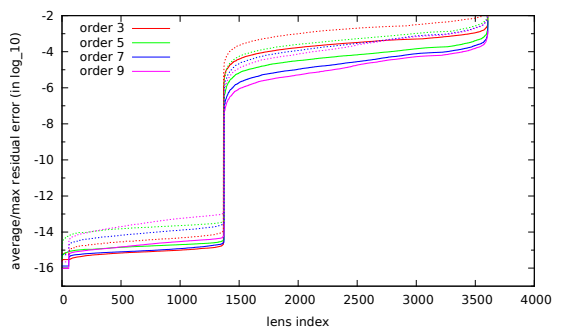

(e) polynomial model for distortion

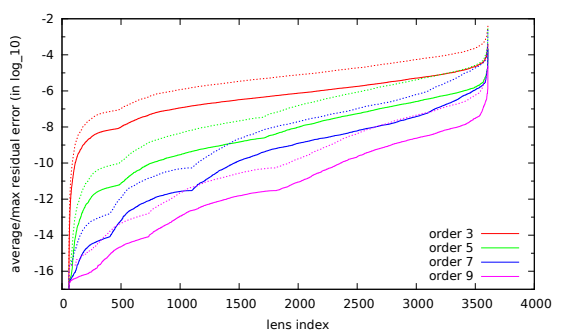

(h) division model for correction

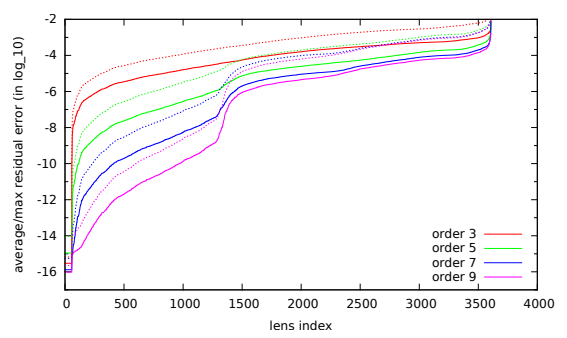

(k) polynomial model for correction

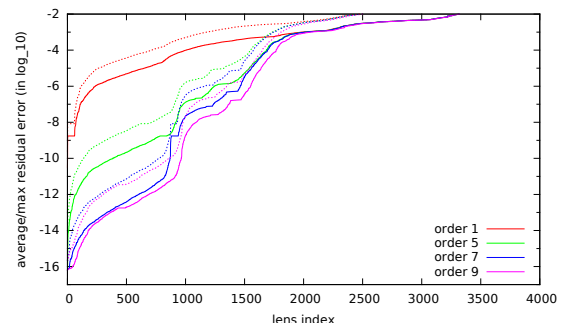

(c) FOV model for distortion

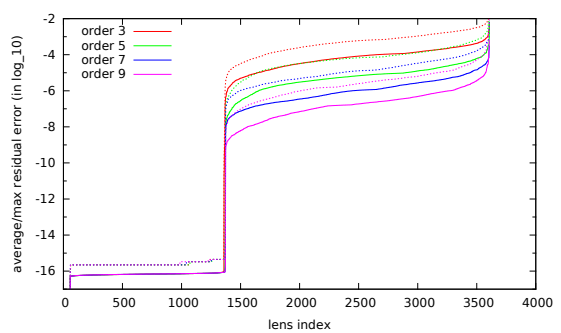

(f) rational model for distortion

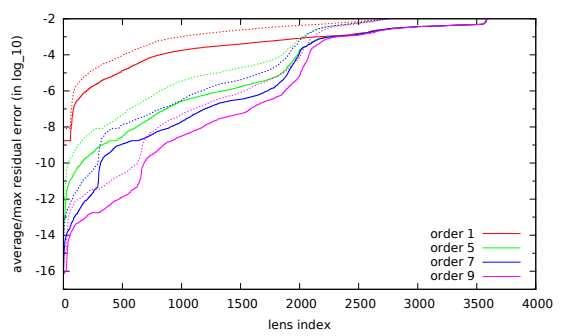

(i) FOV model for correction

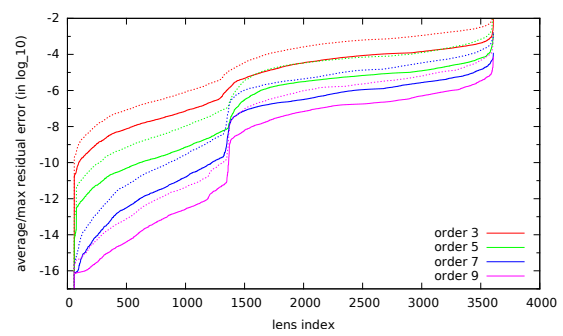

(1) rational model for correction

Fig. 1: The average residual error (dahed line: maximal error) of different models when used to simulate the distortion or its inverse (i.e., the correction) synthesized following the models and the parameters provided by the Lensfun library. The horizontal axis is the lens index (or one lens at different focal lengths) and the vertical axis is the average residual error represented in $\log _{10}$ in the normalized domain. The average residual errors are ordered increasingly. This figure is better viewed electronically in color.

other parameters of distortion models in the incremental LM minimization. Since the initial estimate of $\left(x_{c}, y_{c}\right)$ is already precise, we finally obtained results very similar to the models with $\left(x_{c}, y_{c}\right)$ known and fixed. For the polynomial model and the rational model, since they are invariant to the distortion center, it is not suprising that they fall into the same precision. We do not show these very similar curves to make the paper more concise.

\section{Comparison of radial and division models}

Based on observations of Fitzgibbon [10], the division model is assumed to be more precise than the radial model, at the same number of parameters. This is confirmed by the experiments of Santana-Cedrés et al. [25]. The compared models are infinitely differentiable, with one or two parameters, that is (3) or (4) with: $k_{0}=1, k_{1}=k_{3}=0$, and $k_{2}$, possibly $k_{4}$, as variables. To test them on LensFun models, we need to let $k_{0}$ as variable, as LensFun models are not necessarily close to the identity near the origin. In [25], such radial and 
division models are used to correct the distortion. Therefore, in Figure 2, we measure the precision obtained when trying to inverse the distortions of LensFun models. The division model is indeed more precise than the radial model with the same number of parameters, yet the improvement is marginal. These experiments do not support the observations of the cited articles.

\section{Realistic distortion}

We remark that the lens profile in the LensFun database is only approximatively calibrated: The provided lens distortions are all radially symmetric around the image center. This is the drawback of most commercial software and explains why they correct the distortion at a precision no better than 0.5 pixel. In reality, we are not sure if the real distortion is symmetric around a certain distortion center. The model complemented by the tangential distortion components is perhaps closer to the real distortion [30]:

$$
\begin{aligned}
\bar{x}_{u} & =\bar{x}_{d}+\bar{x}_{d}\left(k_{1} r_{d}^{2}+\cdots+k_{5} r_{d}^{10}\right)+\left[p_{1}\left(r_{d}^{2}+2 \bar{x}_{d}^{2}\right)+2 p_{2} \bar{x}_{d} \bar{y}_{d}\right], \\
\bar{y}_{u} & =\bar{y}_{d}+\bar{y}_{d}\left(k_{1} r_{d}^{2}+\cdots+k_{5} r_{d}^{10}\right)+\left[p_{2}\left(r_{d}^{2}+2 \bar{y}_{d}^{2}\right)+2 p_{1} \bar{x}_{d} \bar{y}_{d}\right],
\end{aligned}
$$

with $p_{1}, p_{2}, p_{3}$ parameters for decentering distortion and $s_{1}$, $s_{2}$ parameters for thin prism distortion. They contribute to both radially symmetric distortion and tangential distortion. This model is often adopted in bundle adjustment algorithms. The parameters calibrated with Lavest et al. algorithm [17] on an EF-S 18-55mm f/3.5-5.6 lens mounted on a Canon EOS 30D camera were used to synthesize the distortion according to (22). The synthesized distortion was then corrected by the different models through incremental LM minimization. The results are shown in Table III. The radial+tangential model has the distortion center fixed at the known distortion center and inverses the distortion synthesized by the same model. The parameters $p_{1}$ and $p_{2}$ were initialized at 0 . For three radially symmetric models, we estimated also the distortion center which makes them best fit the distortion. The polynomial model and the rational model are invariant to the distortion center. It seems that the radial+tangential model can better inverse the distortion (fitting error 0.005 pixels) than the three radially symmetric models (fitting error 0.01 pixels). In fact, since the synthesized distortion follows the above model in 222, it can be inversed by a model of the same family if the tangential component and the radial component satisfy the following constraints (see Fig. 3a):

$$
\begin{aligned}
\Delta t^{\prime} & =\frac{\Delta d \Delta t}{\Delta u}, \\
\Delta r^{\prime} & =\sqrt{\Delta r^{2}+\Delta t^{2}-\Delta t^{\prime 2}} .
\end{aligned}
$$

$\Delta d$ is the distance between the distorted point to the distortion center and $\Delta u$ is the distance between the undistorted point to the distortion center. $\Delta r$ and $\Delta t$ are the radial component and the tangential component of the synthesized distortion. $\Delta r^{\prime}$ and $\Delta t^{\prime}$ are the radial component and the tangential component of inversed distortion. Similarly, the synthesized distortion can also be modeled by the radially symmetric models if the following constraint is satisfied (see Fig. 3b):

$$
\Delta r^{\prime}=\sqrt{\Delta r^{2}+\Delta t^{2}}
$$

where $\left(x_{c}^{\prime}, y_{c}^{\prime}\right)$ denotes the new distortion center for the radially symmetric models.

These constraints are complex and no closed-form formula exists. They can be simulated but not exactly satisfied. This explains why the polynomial and the rational models are even more precise and yield errors on the order of $1 \mathrm{e}-5$ pixels. But we should remember that the distortion is synthesized following (22) and we are not sure if the real distortion can be precisely modeled in this way. We shall confirm the precision and the stability of the models under consideration in the next real experiments. We will see in the real experiments that the radial+tangential models perform sometimes worse than the radially symmetric models. This poses the problem of model selection in practice.

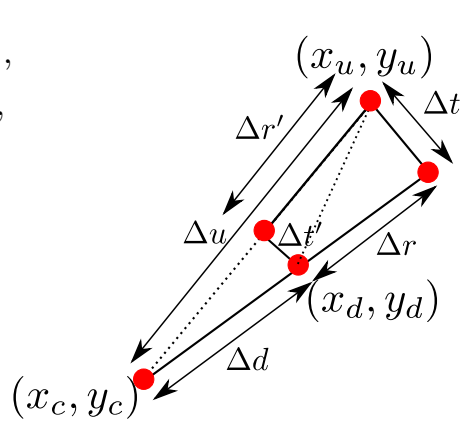

(a)

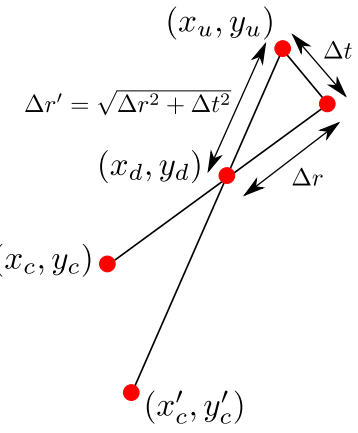

(b)
Fig. 3: The geometric constraint to correct the distortion synthesized by the radial+tangential model. (a) The correction model is the radial+tangential model. The distortion center is fixed and known at $\left(x_{c}, y_{c}\right)$. (b) The correction model is the radially symmetric. The distortion center $\left(x_{c}^{\prime}, y_{c}^{\prime}\right)$ is also estimated.

\begin{tabular}{|c|c|c|c|c|c|c|}
\hline order & R+T & Radial & Division & FOV & Polynomial & Rational \\
\hline 3 & 0.039 & 0.039 & 0.036 & 0.233 & 0.112 & 0.005 \\
\hline 4 & 0.005 & 0.011 & 0.010 & 0.064 & 0.112 & 0.005 \\
\hline 5 & 0.005 & 0.011 & 0.010 & 0.015 & 0.002 & 0.005 \\
\hline 6 & 0.005 & 0.011 & 0.010 & 0.010 & 0.002 & 0.002 \\
\hline 7 & 0.005 & 0.011 & 0.010 & 0.010 & 0.002 & 0.002 \\
\hline 8 & 0.005 & 0.011 & 0.010 & 0.010 & 0.002 & 0.002 \\
\hline 9 & 0.005 & 0.011 & 0.010 & 0.010 & 0.0003 & 0.002 \\
\hline 10 & 0.005 & 0.011 & 0.010 & 0.010 & 0.0003 & 0.002 \\
\hline 11 & 0.005 & 0.011 & 0.010 & 0.010 & 0.00003 & 0.00001 \\
\hline 12 & 0.005 & 0.011 & 0.010 & 0.010 & 0.00003 & 0.00001 \\
\hline
\end{tabular}

TABLE III: The synthesized distortion by one radial+tangential $(\mathrm{R}+\mathrm{T})$ model, corrected by different models. The average residual error is represented in pixels in an image of $1761 \times 1174$ pixels.

\section{REAL DISTORTION FITTING EXPERIMENTS}

We now present real tests to compare the performance of the models for real distortion correction. Our test was based on a non-parametric camera distortion estimation method [11] 

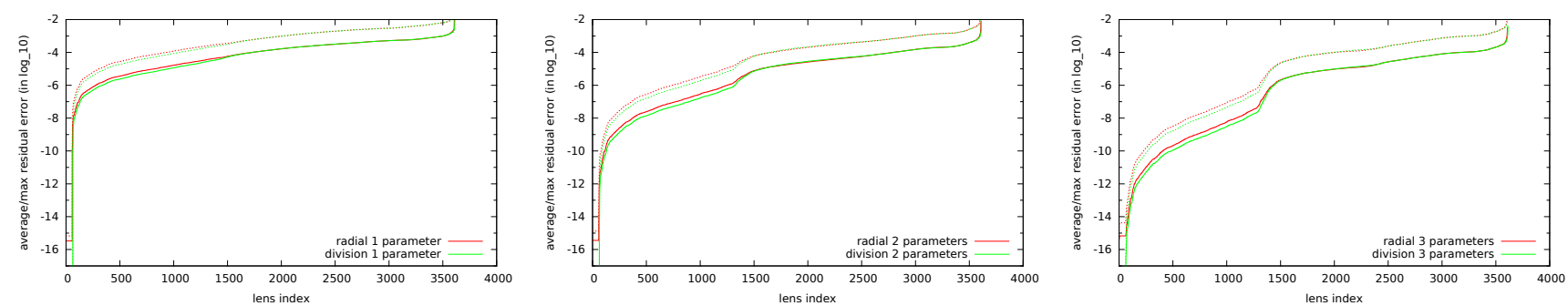

Fig. 2: Comparison of infinitely differentiable radial and division models with few parameters, used for correction. From left to right: one, two, and three parameters in the models. The plain curve represents the average residual error and the dashed curve the maximum error.

but could be performed on any distortion model obtained by blind correction. This method requires a highly textured planar pattern, which is obtained by printing a textured image and pasting it on a very flat object (a thick, heavy and rigid aluminium plate was used in the experiments). Another option is to use a high density grid pattern as in [18]. Two photos of the pattern were taken by a same camera. The distortion is estimated (up to a homography) as the diffeomorphism mapping the original digital pattern to its photograph. The algorithm is summarized below.

1) Take two slightly different photographs of a textured planar pattern (Fig. 4) with a camera whose settings are manually fixed (disable automatic mode);

2) apply the SIFT method [20] between the original digital pattern and two photographs respectively, to obtain matching pairs of points;

3) triangulate and interpolate the SIFT matches from the digital image to two photographs respectively;

4) use a loop validation to eliminate the outlier matches from the digital pattern to one of the two photographs;

5) use a vector filter to remove the few remaining outliers matches from the digital pattern to that photograph;

6) refine the precision of the SIFT matching by correcting each matching point in one image with the local homography estimated from its neighboring matching points;

7) triangulate and interpolate the refined inlier matches to get a dense reverse distortion field from the digital pattern to that photograph;

8) apply the reverse distortion field to any image produced by the same camera to correct the distortion.

For more details please refer to [11].

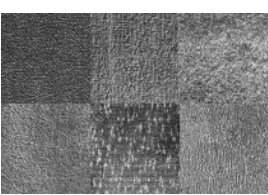

(a)

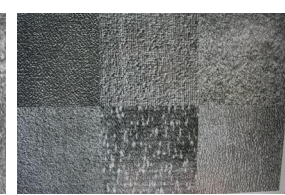

(b)

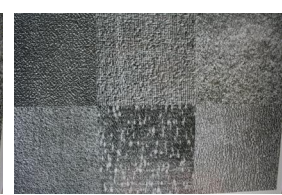

(c)
Fig. 4: (a) digital texture pattern. (b) and (c) two similar photographs of the flat pattern.

The matching pairs delivered by step 6 (about 8000 in our experiments) in the algorithm above do not contain gross outliers and are precise thanks to the local filtering. So we could directly try all the models to fit these matchings. The residual fitting error shows to what extent the models are faithful to a real camera distortion. Under the assumption that the textured pattern is flat, the mapping from the digital pattern to the photo can be modeled as $\mathbf{S D H}$, with $\mathbf{H}$ the homography from the digital pattern to the photo, $\mathcal{D}$ the non-linear lens distortion and $\mathbf{S}$ a diagonal matrix to model the slant of the CCD matrix:

$$
\mathbf{H}=\left(\begin{array}{ccc}
h_{11} & h_{12} & h_{13} \\
h_{21} & h_{22} & h_{23} \\
h_{31} & h_{32} & 1
\end{array}\right), \quad \mathbf{S}=\left(\begin{array}{ccc}
1 & 0 & 0 \\
0 & s & 0 \\
0 & 0 & 1
\end{array}\right)
$$

Since the polynomial and the rational function models can simulate well $\mathbf{H}$ and $\mathbf{S}$, we can use these models to simulate the distortion field without explicitly estimating the homography. Nevertheless, for the radially symmetric models, it is necessary to take into account $\mathbf{H}$ and $\mathbf{S}$ when approximating the distortion. Indeed $\mathbf{H}$ and $\mathbf{S}$ are generally not radially symmetric. Thus, we have 9 additional parameters to estimate, besides the parameters of radially symmetric distortion models and their distortion center. The radial+tangential model is also tried with $\mathbf{H}$ and $\mathbf{S}$ (with the distortion center fixed at the center of image). The polynomial model can again be solved linearly. For all the other models, an incremental LM minimization was used to estimate the distortion center, the distortion parameters, $\mathbf{H}$ and $\mathbf{S}$. The matrix $\mathbf{H}$ was initialized as the homography linearly estimated from the digital pattern to the photo and $s$ is initialized at 1 . The other parameters were initialized in the same way as we did for the synthetic tests. We worked in the normalized image domain to avoid possible numerical problems.

Half the matching pairs were used to estimate the parameters for the different models, and the other half to evaluate the average fitting error. We tried two cameras: a Canon EOS 30D with EF-S 18-55mm lens and a Canon EOS 40D with EF 24-70mm lens. Both extreme focal lengths were tested: $18 \mathrm{~mm}$ and $55 \mathrm{~mm}$ for EF-S $18-55 \mathrm{~mm}$ lens, $24 \mathrm{~mm}$ and $70 \mathrm{~mm}$ for EF $24-70 \mathrm{~mm}$ lens. The results are recapitulated in Table IV. They show that by combining $\mathbf{H}$ and $\mathbf{S}$ to model the inclination between the camera and the pattern, all of the radially symmetric models give almost the same fitting error, which becomes stable with the increase of the model order. The similar estimation of $\mathbf{H}$ and $\mathbf{S}$ indicates that the minimization process is stable. The radial+tangential model is not always better than the radially symmetric models. 
Sometimes, it is even slightly worse $(24 \mathrm{~mm}$ focal length, Table IV].

The polynomial and the rational function models give a stable fitting error, which is smaller than the radially symmetric models. The stability of the fitting error confirms that none of the models suffers from numerical instability or noise fitting. We remark that the residual with the rational function model becomes stable just a little faster than the polynomial model. However, solving the rational function model requires a complex incremental LM minimization, which is a timeconsuming process requiring a good initialization and does not always ensure the convergence to the global minimum, whereas the polynomial model can always be solved linearly.

\begin{tabular}{|c|c|c|c|c|c|c|c|}
\hline & order & $\mathrm{R}+\mathrm{T}$ & $\mathrm{R}$ & $\mathrm{D}$ & $\mathrm{F}$ & $\mathrm{P}$ & $\mathrm{Ra}$ \\
\hline \multirow{5}{*}{$18 \mathrm{~mm}$} & 3 & 0.39 & 0.40 & 0.41 & 0.41 & 1.05 & 0.15 \\
& 4 & 0.10 & 0.18 & 0.17 & 0.39 & 1.12 & 0.05 \\
& 5 & 0.09 & 0.17 & 0.17 & 0.19 & 0.08 & 0.05 \\
& 6 & 0.09 & 0.17 & 0.16 & 0.18 & 0.07 & 0.05 \\
& 7 & 0.09 & 0.17 & 0.17 & 0.17 & 0.04 & 0.04 \\
\hline \hline \multirow{3}{*}{$55 \mathrm{~mm}$} & 3 & 0.08 & 0.07 & 0.07 & 0.07 & 0.07 & 0.05 \\
& 4 & 0.07 & 0.07 & 0.07 & 0.07 & 0.06 & 0.04 \\
& 5 & 0.07 & 0.07 & 0.07 & 0.07 & 0.04 & 0.04 \\
\hline \hline \multirow{3}{*}{$24 \mathrm{~mm}$} & 3 & 0.14 & 0.12 & 0.12 & 0.28 & 0.27 & 0.05 \\
& 4 & 0.12 & 0.11 & 0.10 & 0.15 & 0.23 & 0.04 \\
& 5 & 0.12 & 0.11 & 0.10 & 0.13 & 0.05 & 0.04 \\
\hline \hline \multirow{5}{*}{$70 \mathrm{~mm}$} & 3 & 0.09 & 0.11 & 0.20 & 0.08 & 0.06 & 0.06 \\
& 4 & 0.08 & 0.10 & 0.20 & 0.08 & 0.05 & 0.05 \\
& 5 & 0.08 & 0.09 & 0.20 & 0.08 & 0.04 & 0.04 \\
& 9 & 0.08 & 0.10 & 0.09 & 0.08 & 0.04 & 0.04 \\
\hline
\end{tabular}

TABLE IV: The average fitting error for the considered models for the experiment with cameras Canon EOS 30D and Canon EOS 40D. R+T: radial + tangential model, R: radial model, D: division model, F: FOV model, P: polynomial model and Ra: rational model.

\section{Plumb-Line VALIDATION}

It should be noted that the non-parametric method does not give a ground truth. It is just a non-parametric estimation of the camera distortion, and it is subject to errors. Thus, we needed a more objective evaluation to check the quality of the correction models. To this purpose, a physical frame with tightly stretched opaque fishing strings was built [27]. The physical tension of the strings guarantees a very high straightness. Once the parameters of the models are estimated, a distortion field can be constructed and applied for the distortion correction of images of strings taken by the same camera with the same fixed lens configuration, see Fig. 5. The "average straightness error" is the average distance from the sub-pixel edge points (computed by an improved Canny filter [8]) of the corrected lines to the corresponding regression line (see [27] for more details). Table $\mathrm{V}$ recapitulates the average distance measured on the strings image corrected by different models for the lens we tested. The stabilized straightness error implies again that none of the models has the problem of noise fitting, which guarantees the correction quality and stability. The radial+tangential model does not always perform better than the radially symmetric models and sometimes it is even worse (focal length $24 \mathrm{~mm}$ ). The radially symmetric models with varying distortion center seems enough to fit the real camera distortion precisely when the distortion is not very big. For the big distortion with short focal length $18 \mathrm{~mm}$, the polynomial model and the rational model are twice more precise.

We also remark that the measurement precision provided by the fishing strings is limited by the detection precision of edge points and the small intrinsic oscillation of the fishing strings. The limit measurement precision is around $0.03 \sim$ 0.04 pixels, which appears many times in Table $\mathrm{V}$. This means that the distortion was corrected at approximately the limit precision that could be measured. This fact might also explain why the polynomial and the rational models giving smaller fitting error do not always lead to smaller straightness error. Some other more precise instruments might be able to measure the different errors obtained by the two models.

\begin{tabular}{|c|c|c|c|c|c|c|c|}
\hline & order & $\mathrm{R}+\mathrm{T}$ & $\mathrm{R}$ & $\mathrm{D}$ & $\mathrm{F}$ & $\mathrm{P}$ & $\mathrm{Ra}$ \\
\hline \hline \multirow{5}{*}{$18 \mathrm{~mm}$} & 3 & 0.25 & 0.24 & 0.25 & 0.25 & 0.64 & 0.10 \\
& 4 & 0.06 & 0.06 & 0.07 & 0.24 & 0.63 & 0.03 \\
& 5 & 0.06 & 0.06 & 0.07 & 0.06 & 0.04 & 0.03 \\
& 6 & 0.06 & 0.06 & 0.06 & 0.06 & 0.04 & 0.03 \\
& 7 & 0.06 & 0.06 & 0.06 & 0.06 & 0.03 & 0.03 \\
& 8 & 0.06 & 0.06 & 0.06 & 0.06 & 0.03 & 0.03 \\
\hline \hline \multirow{5}{*}{$55 \mathrm{~mm}$} & 3 & 0.04 & 0.04 & 0.04 & 0.04 & 0.05 & 0.04 \\
& 4 & 0.04 & 0.04 & 0.04 & 0.04 & 0.05 & 0.04 \\
& 5 & 0.04 & 0.04 & 0.04 & 0.04 & 0.04 & 0.04 \\
& 6 & 0.04 & 0.04 & 0.04 & 0.04 & 0.04 & 0.04 \\
& 7 & 0.04 & 0.04 & 0.04 & 0.04 & 0.04 & 0.04 \\
& 8 & 0.04 & 0.04 & 0.04 & 0.04 & 0.04 & 0.04 \\
\hline \hline \multirow{5}{*}{$24 \mathrm{~mm}$} & 3 & 0.09 & 0.06 & 0.06 & 0.18 & 0.14 & 0.03 \\
& 4 & 0.08 & 0.05 & 0.04 & 0.10 & 0.14 & 0.03 \\
& 5 & 0.08 & 0.05 & 0.04 & 0.08 & 0.04 & 0.04 \\
& 6 & 0.08 & 0.05 & 0.04 & 0.08 & 0.04 & 0.04 \\
& 7 & 0.08 & 0.05 & 0.04 & 0.08 & 0.04 & 0.04 \\
\hline \hline \multirow{5}{*}{$70 \mathrm{~mm}$} & 3 & 0.03 & 0.03 & 0.11 & 0.03 & 0.04 & 0.03 \\
& 4 & 0.03 & 0.03 & 0.11 & 0.03 & 0.04 & 0.04 \\
& 5 & 0.03 & 0.03 & 0.11 & 0.03 & 0.04 & 0.04 \\
& 6 & 0.03 & 0.03 & 0.10 & 0.03 & 0.04 & 0.03 \\
& 7 & 0.03 & 0.03 & 0.10 & 0.03 & 0.04 & 0.04 \\
& 8 & 0.03 & 0.03 & 0.10 & 0.03 & 0.04 & 0.04 \\
& 9 & 0.03 & 0.03 & 0.03 & 0.03 & 0.04 & 0.04 \\
\hline
\end{tabular}

TABLE V: The average straightness error for different models for the experiment with cameras Canon EOS 30D and Canon EOS 40D. R+T: radial + tangential model, R: radial model, D: division model, F: FOV model, P: polynomial model and Ra: rational model.

\section{CONCLUSION}

We evaluated and compared the precision of five distortion models. The radially symmetric models are ideal for modeling the radially symmetric distortion, but in practice, the related non-linear optimization raises a numerical risk. These models can be extended by adding tangential distortion components. However, this is not always a good choice and can pose the problem of model selection. The polynomial and the rational models are more universal in the sense that they are invariant to the distortion center and can model realistic non-radially symmetric distortion. The polynomial model requires a little higher degrees than the rational model to achieve the precision on the order of $1 / 100$ pixels. This high degree raises no 


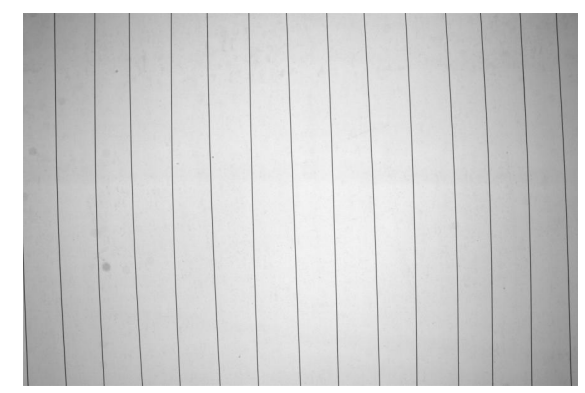

(a) distorted image

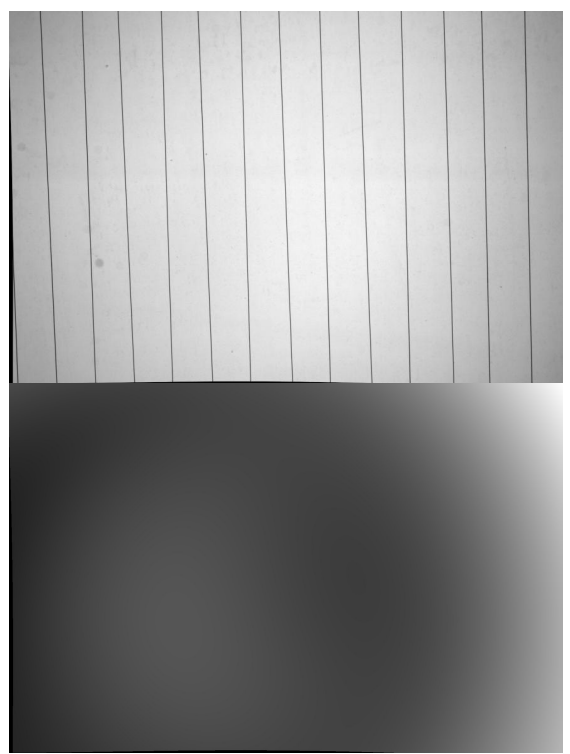

(b) radial model of 12th-order

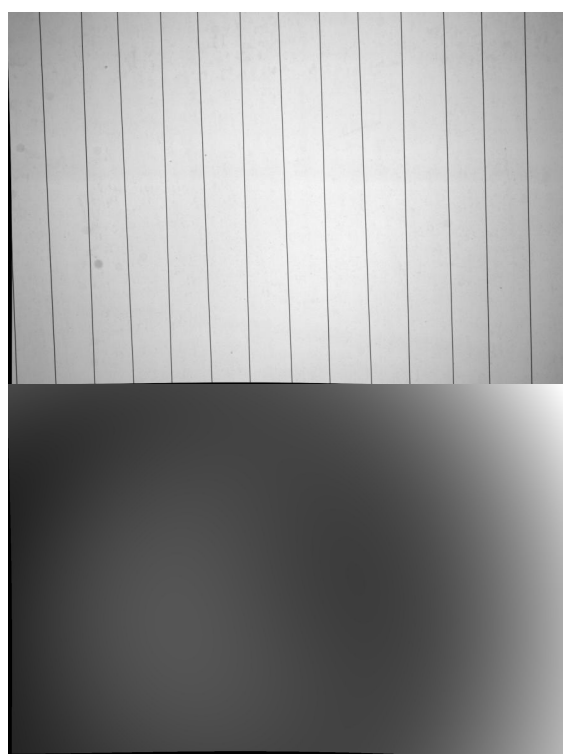

(c) radial+tangential model of 12th-order

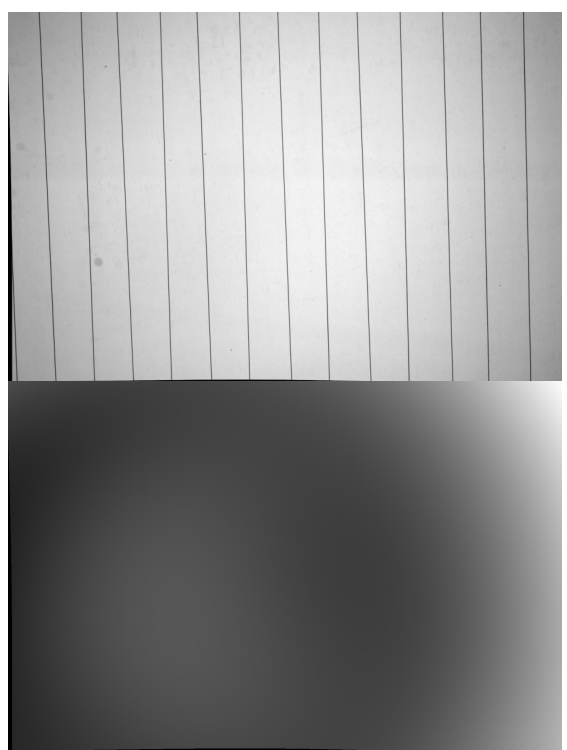

(d) polynomial model of 12th-order

Fig. 5: The experiment with cameras Canon EOS 30D with EF-S 18-55mm lens. The focal length is $18 \mathrm{~mm}$. Top row: distorted image. Second row: corrected images. Third row: distortion field coded as the module of the displacement vector pointing from the digital pattern to the photo. The radial model and the radial+tangential model are with $\mathbf{H}$ and $\mathbf{S}$ in (24). In fact, the results of all the tested parametric models are visually identical, according to the accuracy shown in Tables $[\mathrm{IV}$ and $\mathrm{V}$

computational problem for the polynomial model, which can be solved linearly after a correct conditioning.

The polynomial and the rational models are not adapted to global bundle adjustment, where the internal/external parameters and the distortion model are estimated simultaneously. The distortion correction must be dealt with as an independent and previous step to camera calibration [24]. All the models are able to correct the distortion at sub-pixel precision better than 0.1 pixel and the polynomial and the rational models seem to perform better when the distortion is large. The polynomial model raises less numerical risk since it can be solved linearly. The only requirement is to dispose of many more control points than the number of parameters. This might be an objection when using over-simple calibration patterns. In our experiments the number of control points (about 4000) was far higher, about 60 times the number of polynomial coefficients $((7+1) \cdot(7+2)=72$ for a 7 th-order polynomial). Clearly, this also entails two methodological changes: first that the distortion should be corrected independently and previously to projective calibration. Second, that calibration patterns should contain a higher than usual number of control points (more than 500). With the current image resolution in most cameras, this is no longer a restriction. With these provisions, the polynomial model is clearly the best model in terms of high precision and of ease of evaluation.

\section{ACKNOWLEDGMENTS}

This work was supported by Agence Nationale de la Recherche ANR-09-CORD-003 (Callisto project), Direction Générale de l'Armement ANR-12-ASTR-0035 (STEREO project), Centre National d'Etudes Spatiales (MISS project), Office of Naval research under grant N00014-97-1-0839, and European Research Council, advanced grant "Twelve labours".

\section{REFERENCES}

[1] L. Alvarez, L. Gómez, and J. R. Sendra. An algebraic approach to lens distortion by line rectification. Journal of Mathematical Imaging and Vision, 35(1):36-50, 2009.

[2] L. Alvarez, L. Gomez, and J. R. Sendra. Algebraic lens distortion model estimation. Image Processing On Line, 1, 2010.

[3] D.C. Brown. Close-range camera calibration. Photogrammetric Engieering, 37:855-866, 1971 . 
[4] M. Byrod, Z. Kukelova, K. Josephson, T. Pajdla, and K. Astrom. Fast and robust numerical solutions to minimal problems for cameras with radial distortion. Computer Vision and Image Understanding, 114(2):18, 2008.

[5] T. A. Clarke, X. Wang, and J. G. Fryer. The principal point and CCD cameras. The Photogrammetric Record, 16(92):293-312, 2003.

[6] D. Claus and A. W. Fitzgibbon. A plumbline constraint for the rational function lens distortion model. BMVC, pages 99-108, 2005.

[7] D. Claus and A. W. Fitzgibbon. A rational function lens distortion model for general cameras. CVPR, 1:213-219, 2005.

[8] F. Devernay. A non-maxima suppression method for edge detection with sub-pixel accuracy. Technical Report 2724, INRIA, 1995.

[9] F. Devernay and O. Faugeras. Straight lines have to be straight. Mach. Vision Appli., 13:14-24, 2001.

[10] A. W. Fitzgibbon. Simultaneous linear estimation of multiple view geometry and lens distortion. CVPR, 1:125-132, 2001.

[11] R. Grompone von Gioi, P. Monasse, J.-M. Morel, and Z. Tang. Towards high-precision lens distortion correction. ICIP, pages 4237-4240, 2010.

[12] R. Hartley and S.S. Kang. Parameter-free radial distortion correction with centre of distortion estimation. TPAMI, 29(9), aug 2007.

[13] R. I. Hartley and T. Saxena. The cubic rational polynomial camera model. Proc. DARPA Image Understanding Workshop, pages 649-653, 1997.

[14] R.I Hartley and A. Zisserman. Multiple View Geometry in Computer Vision. Cambridge Univ. Press, 2000.

[15] E. Kilpelä. Compensation of systematic errors of image and model coordinates. Photogrammetria, 37(1):15-44, 1980.

[16] Z. Kukelova and T. Pajdla. A minimal solution to the autocalibration of radial distortion. CVPR, page 17, 2007.

[17] J. Lavest, M. Viala, and M. Dhome. Do we really need accurate calibration pattern to achieve a reliable camera calibration? ECCV, 1:158-174, 1998

[18] S. Lee, B. Kim, J. Lee, and J. Sasian. Accurate determination of distortion for smartphone cameras. Applied Optics, 53:H1-H6, 2014.

[19] H. Li and R. Hartley. A non-iterative method for correcting lens distortion from nine point correspondences. OmniVis, 2005.

[20] David G Lowe. Distinctive image features from scale-invariant keypoints. IJCV, 60(2):91-110, 2004.

[21] T. Pajdla, Z. Kukelova, and M. Bujnak. Automatic generator of minimal problem solvers. ECCV, pages 302-315, 2008.

[22] T. Pajdla, T. Werner, and V. Hlavac. Correcting radial lens distortion without knowledge of 3-D structure. Research Report, Czech Technical University, 1997.

[23] B. Prescott and G. F. Mclean. Line-based correction of radial lens distortion. Graphical Models and Image Processing, 59:39-47, 1997.

[24] V. Rudakova and P. Monasse. Camera matrix calibration using circular control points and separate correction of the geometric distortion field. In Canadian Conference on Computer and Robot Vision (CRV), pages 195-202. IEEE, 2014.

[25] D. Santana-Cedrés, L. Gómez, M. Alemán-Flores, A. Salgado, J. Esclarín, L. Mazorra, and L. Álvarez. An iterative optimization algorithm for lens distortion correction using two-parameter models. Image Processing On Line, 6:326-364, 2016.

[26] G. P. Stein. Lens distortion calibration using point correspondences. CVPR, pages 602-608, 1997.

[27] Z. Tang, R. Grompone von Gioi, P. Monasse, and J.-M. Morel. Highprecision camera distortion measurements with a "calibration harp". Journal of the Optical Society of America A, 29(10):2134-2143, 2012.

[28] M. Thirthala and S. Pollefeys. Multi-view geometry of 1d radial cameras and its application to omnidirectional camera calibration. ICCV, pages $1539-1546,2005$.

[29] R. Y. Tsai. A versatile camera calibration technique for high-accuracy 3D machine vision metrology using off-the-shelf TV cameras and lenses. IEEE Journal of Robotics and Automation, Vol. RA-3, 1987.

[30] J. Weng, P. Cohen, and M. Herniou. Camera calibration with distortion models and accuracy evaluation. TPAMI, 14(10):965-980, 1992.

[31] Z. Zhang. On the epipolar geometry between two images with lens distortion. ICPR, pages 407-411, 1996.

[32] Z. Zhang. A flexible new technique for camera calibration. ICCV, pages 663-673, September 1999.

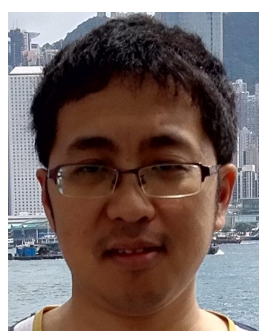

Zhongwei Tang received the B.Sc. degree in Electronic Engineering in Fudan University, China (2003). He then obtained the M.Sc. (2007) and Ph.D. (2011) in Applied Mathematics from the École Normale Supérieure de Cachan, France. He also obtained an Engineer Degree (2006) in ENSEEIHTINP (Institut National Polytechnique), France. He was a postdoctoral research associate at the University of Minnesota, Duke University and École des Ponts ParisTech, France (2012-2013). Now he works for Huawei Technologies in Shanghai and leads the efforts of the $R \& D$ related to the depth sensing for its latest mobile phone.

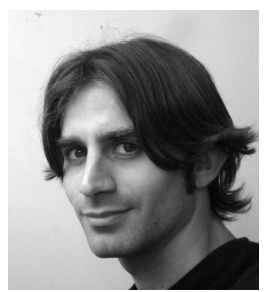

Rafael Grompone von Gioi received the B.Sc. degree in Electrical Engineering from the Universidad de la República, Uruguay (2004). He then obtained the M.Sc. (2006) and Ph.D. (2010) in Applied Mathematics from the École Normale Supérieure de Cachan, France. He is currently a post-doc at the École Normale Supérieure de Cachan.

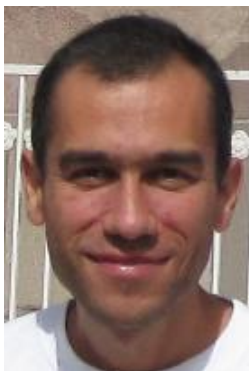

Pascal Monasse received a Ph.D. degree in applied mathematics from the University Paris-Dauphine in 2000 and the Habilitation from École Normale Supérieure de Cachan in 2013. He previously got an engineering degree from École Nationale des Ponts et Chaussées in 1995. He worked as a junior then senior researcher at Cognitech, Inc. in Pasadena, California, on image and video processing from 2001 to 2007. The software product PixL2GPS, of which he was the main developper and project leader while working at Cognitech, received in 2010 the American Technology Awards "The Termans" from the TechAmerica Foundation. He joined the Imagine group at École des Ponts ParisTech as a research scientist in 2008 and is a member of Laboratoire d'Informatique Gaspard Monge (LIGM), Université Paris-Est Marne-la-Vallée. His current research interests include computer vision, stereo reconstruction and mathematical morphology.

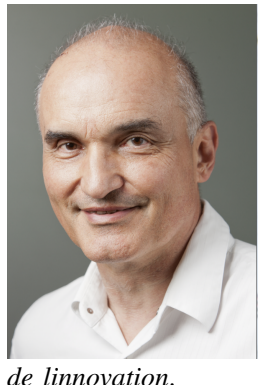

Jean-Michel Morel received the $\mathrm{PhD}$ degree in applied mathematics from University Pierre et Marie Curie, Paris, France in 1980. He started his career in 1979 as assistant professor in Marseille Luminy, then moved in 1984 to University Paris-Dauphine where he was promoted professor in 1992. He is Professor of Applied Mathematics at the École Normale Supérieure de Cachan since 1997. His research is focused on the mathematical analysis of image processing. He is a 2015 laureate of the Longuet-Higgins prize and of the CNRS médaille 